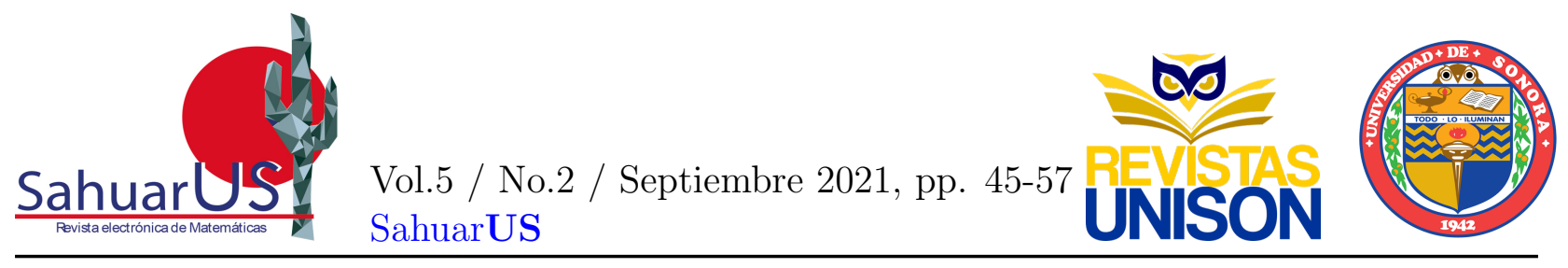

\title{
EvA-Web: Una herramienta para evaluar la accesibilidad de sitios web
}

\author{
Gabriel García-Mireles ${ }^{1}$, Angélica María Maldonado Vásquez ${ }^{2}$ y Miguel Ehécatl \\ Morales-Trujillo ${ }^{3}$ \\ Departamento de Matemáticas, Universidad de Sonora ${ }^{1,2}$ \\ Universidad de Canterbury ${ }^{3}$ \\ 1' gabriel.garcia@unison.mx, ${ }^{2}$ a207209239@unison.mx, ${ }^{3}$ miguel.morales@canterbury.ac.nz
}

\section{Resumen}

En la actualidad, las personas dependen en gran medida de las tecnologías de la información para realizar su trabajo, acceder contenidos para la formación académica o entretenimiento. En este contexto, los sitios web permiten compartir la información con una amplia variedad de usuarios. Para lograr que los contenidos sean percibidos por todas las personas, incluyendo aquellas que tienen alguna discapacidad, se requiere que los sitios web sean accesibles. El término accesibilidad denota el grado en el cual un sistema puede ser usado por personas con el más amplio rango de capacidades. El objetivo de este trabajo, por tanto, es presentar EvA-Web (Evaluación de la Accesibilidad-Web) como una herramienta que apoya a los desarrolladores de software en la identificación de las barreras a la accesibilidad que se encuentran en los sitios web que diseñan o programan. El prototipo de esta herramienta se desarrolló tomando en cuenta las guías de accesibilidad para el contenido web (Web Content Accessibility Guidelines) WCAG 2.0. Además, presentamos los resultados de la validación del prototipo de EvA-Web en donde se evaluó un sitio web y se identificaron barreras a la accesibilidad. La herramienta EvA-Web es uno de los productos generados de una tesis realizada por una estudiante de la licenciatura en Ciencias de la Computación que se ofrece en la Universidad de Sonora.

Palabras Clave: Accesibilidad, Herramienta de evaluación, Sitios web, WCAG 2.0, Web Content Accessibility Guidelines.

DOI: $10.36788 /$ sah.v5i2.103

Recibido 2 de marzo de 2020

Aceptado 8 de septiembre de 2021 


\section{Introducción}

Muchas de las experiencias cotidianas de los individuos están relacionadas con el acceso a dispositivos digitales, computadoras y teléfonos celulares, en donde la web se convierte en una vía primaria para acceder a los contenidos. Desde sus inicios, la web se conceptualizó como una herramienta de trabajo para todas las personas, cualquiera que sea su hardware, software, lenguaje, localización geográfica o nivel de habilidad o capacidad (Henry and McGee, 2018). Para que la web logre su objetivo de compartir información entre distintas personas, ésta debe ser accesible.

En este trabajo estamos interesados en proporcionar accesibilidad a través de la web a todas las personas. Accesibilidad significa que los "sitios web, herramientas, y tecnologías se diseñan y desarrollan para que las personas con discapacidades las puedan usar... [es decir], puedan percibir, entender, navegar, .., [y] contribuir a la web" (W3C Working Group, 2018). Dado que las personas que usan la web pueden tener distintos niveles de capacidades físicas o cognitivas, es importante considerar sus limitaciones en el diseño de sitios web. En particular, el término "discapacidad" se refiere a deficiencias, limitaciones de actividad y restricciones para la participación de los individuos (World Health Organization, 2011).

Se estima que el $15 \%$ de la población, conformada por personas mayores de 14 años, vive con alguna discapacidad (World Health Organization, 2011). De éstas, alrededor del $4 \%$ tienen una discapacidad grave, e.g., ceguera, depresión grave o tetraplejía. Además, los datos estadísticos muestran que el número de personas con discapacidad está creciendo debido al envejecimiento de la población y enfermedades crónicas como la diabetes, enfermedades cardiovasculares y trastornos mentales (World Health Organization, 2011). En el caso de México, el INEGI (Instituto Nacional de Estadística y Geografía (México), 2016) informa que el $13 \%$ de la población total tiene alguna limitación en sus capacidades y la mitad de ellos están en el grupo de edad de 30 a 59 años (p. 97). Las discapacidades de mayor prevalencia son las motrices y visuales, las cuales fueron reportadas por más de la mitad de las personas con discapacidad (Instituto Nacional de Estadística y Geografía (México), 2016).

Las personas con discapacidad enfrentan varios obstáculos para valerse por sí mismas cuando necesitan acceder los contenidos digitales en la web. Entre ellos, se encuentran la insuficiente calidad en los servicios provistos por instituciones gubernamentales y no gubernamentales, la falta de accesibilidad a la información y servicios básicos como internet, la falta de la implementación de criterios de accesibilidad en sitios web, entre otros Acosta et al., 2018). Las barreras a la accesibilidad desalientan a las personas con discapacidad a buscar trabajo, recibir atención sanitaria o educación (Instituto Nacional de Estadística y Geografía (México), 2016). La falta de acceso igualitario a los servicios en internet ha resultado en la aparición de términos relacionados con la exclusión, como infoexclusión, exclusión digital y exclusión social (Agangiba and Kabanda, 2017). Es importante para las personas con discapacidad estar integradas en la sociedad digital en donde ellas puedan disfrutar de acceso igualitario a las oportunidades que ofrece la web, lo que podría contribuir a su desarrollo personal al fomentar su independencia, sentimientos de pertenencia y su actualización (Agangiba and Kabanda, 2017). 
Para mejorar la accesibilidad de la web, los desarrolladores de sitios y aplicaciones web podrían aplicar un conjunto de recomendaciones para que los contenidos de las páginas web sean fácilmente codificados e interpretados por tecnologías de asistencia a la discapacidad (e.g., lectores de pantallas). Este es un primer paso para facilitar el acceso a los contenidos web. Para ejecutarlo, es importante conocer y aplicar las guías de accesibilidad disponibles (e.g. Web Content Accesiblity Guidelines (WCAG 2.0)) (W3C Working Group, 2008). El propósito de estas guías es proporcionar el control al usuario sobre los eventos que se presentan en la pantalla y facilitarle la interacción con los sitios web a través del uso de tecnologías de asistencia. Aunque estas guías ya tienen algún tiempo publicadas, aún existen muchos sitios web que presentan barreras de accesibilidad (Laitano, 2015; Power et al., 2012).

Dada la necesidad de mejorar la accesibilidad de sitios web, el objetivo de este artículo es presentar el prototipo EvA-Web, herramienta que permite determinar en qué medida se consideraron las técnicas para mejorar la accesibilidad de páginas web escritas en código (Hypertext Markup Language) HTML 5.0. Por tanto, la sección 2 describe brevemente la guía de accesibilidad WCAG 2.0 mientras que la sección 3 presenta algunos elementos del diseño de la herramienta EvA-Web. Además, la sección 4 muestra la estrategia de validación del prototipo. Finalmente, en la sección 5 presentamos las conclusiones y trabajo futuro.

\section{Guías de la WCAG 2.0}

La WCAG 2.0 (W3C Working Group, 2008) es un guía que permite mejorar la accesibilidad del contenido y de las aplicaciones web. Ha sido desarrollada por el World Wide Web Consortium (W3C), y se puede aplicar tanto al contenido basado en HTML como aquel basado en multimedia, JavaScript, PDF, entre otros. La WCAG 2.0 cubre un amplio rango de características para que el contenido web pueda ser accesible para personas con capacidades diferentes por medio del uso de tecnologías de asistencia, tales como los lectores de pantallas o las impresoras braille.

La guía WCAG 2.0 se estructura en los niveles jerárquicos de principios, objetivos, criterios y técnicas. En el nivel más alto de la jerarquía se encuentra los principios, los cuales describen los fundamentos de la accesibilidad web. Los principios básicos son los de percepción, operación, comprensión y robustez. El principio de percepción se enfoca en encontrar una forma de presentar la información de tal manera que sea accesible por alguno de los sentidos del usuario. Se considera que el principio de operación se ha aplicado en el sitio web cuando el usuario tiene control sobre la navegación según sus capacidades. Cuando el usuario comprende el contenido así como la operación de la interfaz de usuario, entonces se ha aplicado el principio de comprensión. Finalmente, el principio de robustez trata de que el contenido de la página web sea accesible de manera confiable a través de distintas tecnologías de asistencia, las cuales evolucionan a través del tiempo (W3C Working Group, 2008).

Cada principio está asociado a un conjunto de objetivos que permiten crear contenido web accesible (Figura 1). Además, cada objetivo está relacionado con un conjunto de criterios de éxito que deben ser logrados para estar en conformidad con la guía WCAG 2.0. Estos criterios de éxito están escritos de tal manera que pueden probarse objetivamente en distintas 


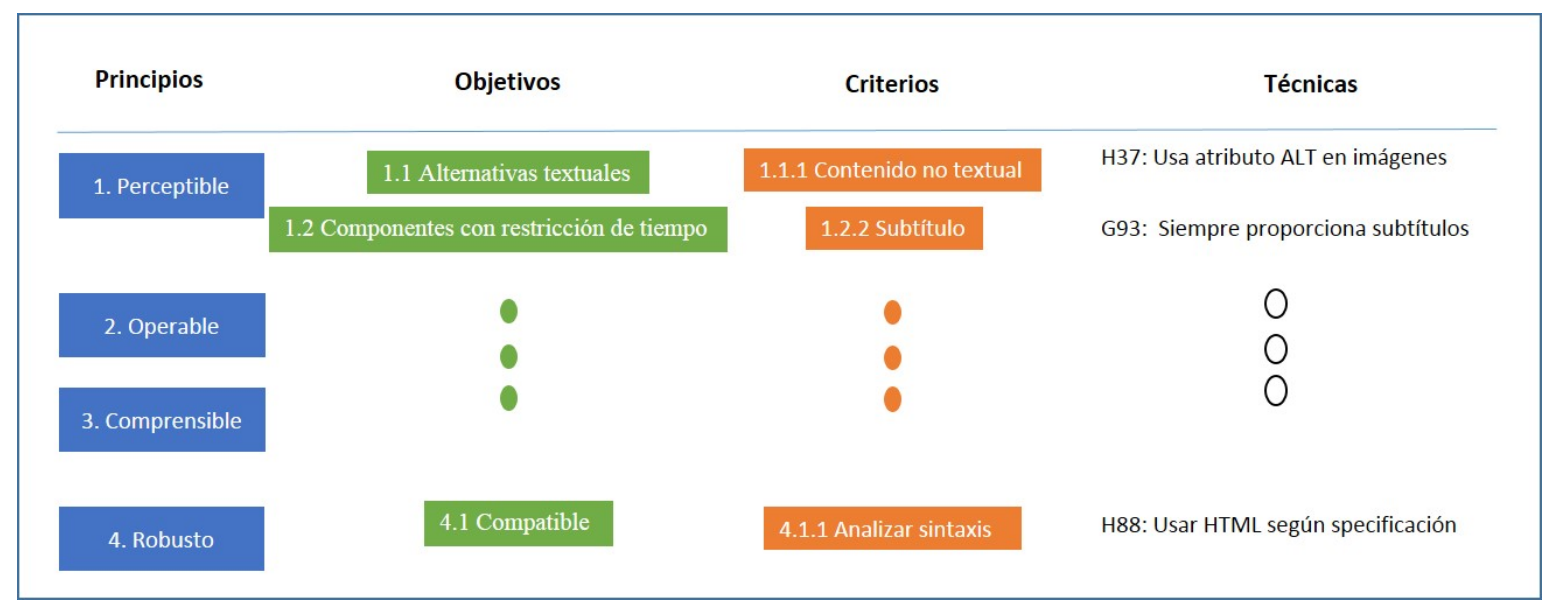

Figura 1: Principios de la WCAG 2.0 y algunos objetivos, criterios y técnicas.

tecnologías de desarrollo de aplicaciones web. La evaluación de los criterios de éxito puede ser realizada con una herramienta de software automatizada, con la intervención de usuarios finales y por expertos en la accesibilidad. Para cada objetivo, la WCAG 2.0 presenta un conjunto de prácticas para evaluar el grado de accesibilidad de algún elemento de la página web tomando en cuenta la tecnología con la que fue creada (e.g., HTML, CSS, JavaScript).

Los criterios de éxito son la base para establecer la conformidad con la WCAG 2.0 de la página web bajo evaluación. La evaluación se realiza en tres niveles A, AA y AAA. En donde el nivel más bajo es $\mathrm{A}$, y éste considera los criterios esenciales para que una tecnología de asistencia pueda hacer el contenido accesible. Cada criterio de éxito tiene asociado un conjunto de técnicas informativas que indican cómo se pueden implementar. Algunas técnicas son específicas a la tecnología usada para construir las páginas web (e.g., HTML).

En este trabajo nos centramos en estudiar las técnicas de accesibilidad dirigidas al código HTML 5.0. El conjunto de técnicas lo denominamos técnicas $\mathrm{H}+$ número. Éstas las clasificamos en ocho categorías: enlace, imagen, página, tablas, estructura, formulario, párrafo y otros. Cada técnica está documentada en la guía de la WCAG 2.0 con base en la prueba que debe realizarse en el contenido HTML (W3C Working Group, 2012). Por ejemplo, la técnica H37 requiere que la etiqueta IMG contenga el atributo ALT que describa en texto la información relevante de la imagen (siempre que la imagen no sea sólo un adorno de la página).

Para desarrollar el prototipo de la herramienta EvA-Web, revisamos artículos científicos, publicados en los últimos diez años, que señalan las características deseables de las herramientas de evaluación de la accesibilidad. En general, se recomienda que estas herramientas informen de las guías, estándares o normativas de accesibilidad que han sido implementadas, especifiquen las técnicas de accesibilidad aplicadas y sus resultados, así como que determinen en qué medida la página web evaluada alcanzó el nivel A, AA o AAA de accesibilidad. Por otra parte, la información mínima de entrada de estas herramientas es la dirección URL del sitio web que se pretende evaluar.

Además, para comprender cómo se aplican las técnicas de evaluación de accesibilidad recomendadas por la WCAG 2.0, analizamos algunas herramientas disponibles en línea como 
Cynthia Says (http://www.cynthiasays.com/), AChecker (https://achecker.ca/checker/ index.php), Taw (https://www.tawdis.net/), Examinator (http://examinator.ws/) y Wave (https://wave. webaim.org/). Estas herramientas solicitan al usuario que introduzca la URL de la página web y que seleccione la guía de accesibilidad por evaluar. En el caso de los informes generados por estas herramientas, encontramos diferencias en el nivel de detalle en que se presenta los resultados de evaluar las técnicas de accesibilidad y el grado en que cada una de las técnicas evaluadas fue satisfecha.

Para determinar la consistencia en los informes generados por las herramientas anteriormente mencionadas, realizamos una evaluación de la accesibilidad, realizada en noviembre de 2019, para determinar en qué grado la página inicial del sitio web de la licenciatura en Ciencias de la Computación de la Universidad de Sonora (http://cc.uson.mx/) es accesible. La razón de haber seleccionado esta página es que difunde información general sobre un programa educativo que debería de llegar a todos los interesados en estudiar dicha licenciatura. La Tabla 1 presenta los resultados parciales de la evaluación, mostrando sólo cinco técnicas de las 31 evaluadas.

Tabla 1: Resultados parciales de la evaluación de la accesibilidad del código HTML con la herramientas seleccionadas.

\begin{tabular}{llllll}
\hline Técnica $H$ & Wave & Taw & Examinator & Cynthia Says & AChecker \\
\hline H2 & 0 & 0 & 2 errores & 6 errores & 0 \\
H4 & 0 & 1 advertencia & 0 & 1 advertencia & 0 \\
H37 & 2 errores & 0 & 0 & 2 errores & 8 advertencias \\
H24 & 0 & 0 & 0 & 0 & 0 \\
H35 & 0 & 0 & 0 & 0 & 0 \\
\hline
\end{tabular}

Como se muestra en la Tabla 1, existen inconsistencias en los resultados reportados por cada una de las herramientas. Esta variación se debe a las diferentes implementaciones de las técnicas $\mathrm{H}$, según el contexto de la página web que haya considerado el programador de la herramienta. Como resultado, la clasificación de los hallazgos es distinta. Algunos tipos de errores encontrados por las herramientas están relacionados con más de una técnica $\mathrm{H}, \mathrm{y}$ eso complica la identificación de la técnica a la cual pertenecen los resultados. Además, un defecto común de las herramientas, es que no reportan el número de renglón correcto en que se encuentra el código que la técnica evalúa.

De hecho, nuestros resultados de este ejercicio de evaluación son consistentes con otros que reportan el desempeño de herramientas de evaluación de accesibilidad. Por ejemplo, Vigo et al. (Vigo et al. 2013) analizaron seis herramientas de evaluación automática de accesibilidad y encontraron diferencias en cuanto a salidas correctas ( $66 \%$ al $96 \%$ ), cobertura de los criterios de éxito (menor al $50 \%$ ) y completitud de la herramienta en donde, en el mejor de los casos, sólo se identifican 4 de 10 violaciones a la accesibilidad. Se cree que estas herramientas están diseñadas para capturar los problemas de accesibilidad más comunes y que poco esfuerzo 
se dedica al desarrollo de componentes que identifiquen violaciones a la accesibilidad menos frecuentes (Vigo et al., 2013).

\section{Herramienta EvA-Web}

Con base en el análisis de herramientas de evaluación automática de la accesiblidad de páginas web y las recomendaciones de investigadores en este campo, se decidió implementar EvA-Web. Las técnicas para evaluar la accesibilidad de páginas web, codificadas en HTML 5.0 se clasificaron de acuerdo al elemento del lenguaje HTML que abordan. La Tabla 2 presenta las 56 técnicas implementas de la WCAG 2.0 (W3C Working Group, 2016).

La clasificación considera las categorías enlace (link), imagen, páginas HTML, tablas, estructura del contenido, formulario, párrafo y otros. Aunque algunas técnicas $\mathrm{H}$ pueden aplicarse a más de un elemento HTML, se decidió en este prototipo asignarlas a una sola categoría. Por ejemplo, en la categoría imagen se encuentran las técnicas H37 y H67. La primera de ellas evalúa que cada elemento IMG tenga el atributo ALT, y que éste describa el contenido de la imagen. En el caso de la técnica H67, se evalúa que cada elemento IMG, que contenga una imagen decorativa, el atributo ALT debe tener valor nulo.

Tabla 2: Técnicas H clasificadas por categoría.

\begin{tabular}{ll}
\hline Categoría & Técnicas \\
\hline Enlace & $\mathrm{H} 2, \mathrm{H} 77, \mathrm{H} 78, \mathrm{H} 79, \mathrm{H} 80, \mathrm{H} 30, \mathrm{H} 83, \mathrm{H} 59, \mathrm{H} 33, \mathrm{H} 97$ \\
Imagen & $\mathrm{H} 24, \mathrm{H} 37, \mathrm{H} 36, \mathrm{H} 67, \mathrm{H} 46$ \\
Página & $\mathrm{H} 25, \mathrm{H} 71, \mathrm{H} 64, \mathrm{H} 57, \mathrm{H} 58, \mathrm{H} 40, \mathrm{H} 93, \mathrm{H} 76, \mathrm{H} 60, \mathrm{H} 59, \mathrm{H} 42, \mathrm{H} 81$ \\
Tablas & $\mathrm{H} 39, \mathrm{H} 63, \mathrm{H} 43, \mathrm{H} 73, \mathrm{H} 51$ \\
Estructura & $\mathrm{H} 4, \mathrm{H} 53, \mathrm{H} 46, \mathrm{H} 70$ \\
Formulario & $\mathrm{H} 32, \mathrm{H} 36, \mathrm{H} 84, \mathrm{H} 44, \mathrm{H} 85, \mathrm{H} 89, \mathrm{H} 90, \mathrm{H} 91, \mathrm{H} 65, \mathrm{H} 35$ \\
Párrafo & $\mathrm{H} 62, \mathrm{H} 28, \mathrm{H} 49, \mathrm{H} 54, \mathrm{H} 56, \mathrm{H} 34$ \\
Otros & $\mathrm{H} 86, \mathrm{H} 88, \mathrm{H} 95, \mathrm{H} 96$ \\
\hline
\end{tabular}

\subsection{Diagrama de casos de uso de EvA-Web}

Los requisitos de usuario que se abordan en el prototipo de la herramienta EvA-Web se describen a través del modelo de casos de uso (Figura 21). La herramienta está dirigida a desarrolladores de software y pueden ejecutar cinco funciones principales de EvA-Web. En general, el desarrollador de software puede obtener información sobre la guía de accesibilidad implementada, obtener información de las características de la herramienta EvA-Web, así como determinar los enlaces por evaluar del sitio web, seleccionar técnicas de accesibilidad y evaluar el sitio web. 


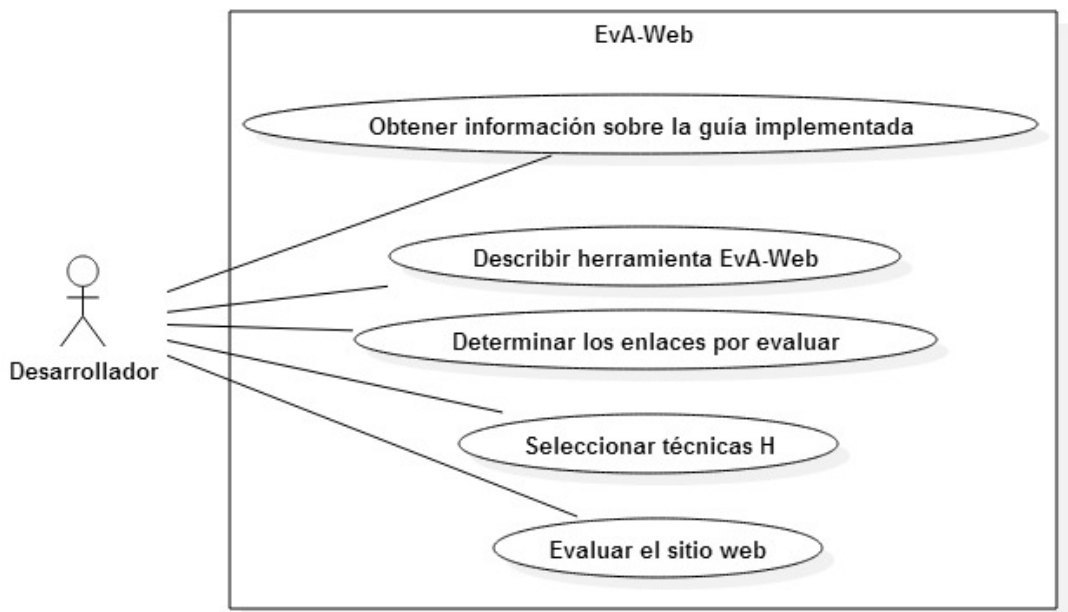

Figura 2: Diagrama de casos de uso de la herramienta EvA-Web.

\subsection{Formularios e informes de EvA-Web}

La página de inicio de EvA-Web (Figura 3) presenta dos tablas, una de ellas muestra los enlaces para acceder la información de la guía implementada y de la herramienta Eva-Web; y la otra, un formulario en que se introduce el enlace del sitio web que se desea evaluar. Además, el desarrollador de software puede indicar si desea evaluar solo una página, o varias, del sitio web.

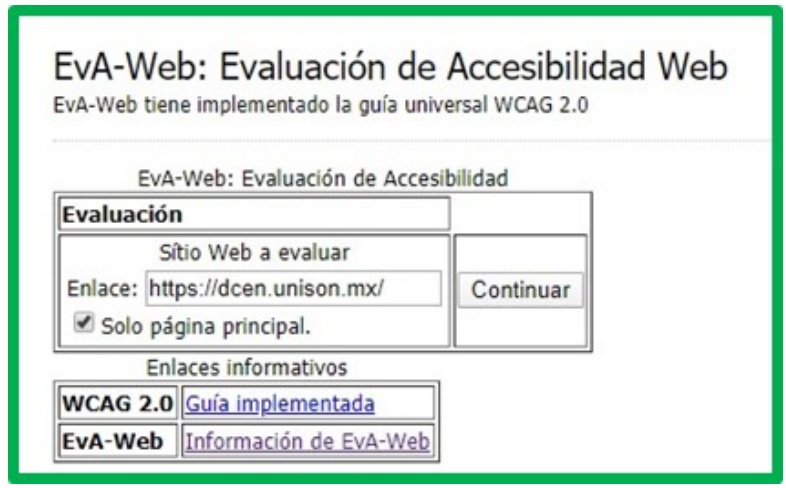

Figura 3: Página de inicio de EvA-Web.

En el caso que se evalúe únicamente el enlace que se ha puesto en el formulario, se requiere confirmar la casilla de verificación "Sólo página principal" antes de hacer clic en el botón "Continuar" (Figura 3). EvA-Web presenta los datos de la evaluación: URL de la página web que será evaluada y el identificador de la evaluación. Si el desarrollador decidió evaluar más de una página del sitio web, aparecerá el formulario con los enlaces encontrados por el EvA-Web Crawler (Figura 44). Este es un formulario que, para cada página del sitio web recuperada, presenta una casilla de verificación la cual permite al desarrollador seleccionar varios páginas o todas ellas.

Tras haber seleccionado los enlaces, se eligen las técnicas H por evaluar. Se abre el menú 


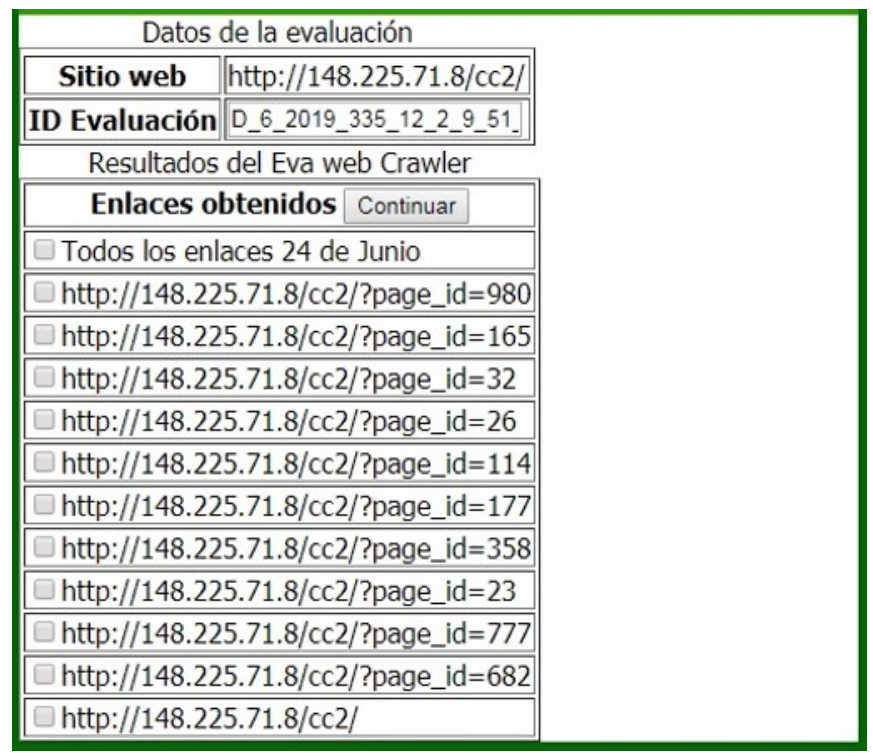

Figura 4: Formulario para la selección de páginas web por evaluar.

de acordeón y se puede elegir una técnica al marcar la casilla de verificación correspondiente. Además, se presentan casillas de verificación para seleccionar categorías enteras o todas las técnicas implementadas en EvA-Web (Figura 5).

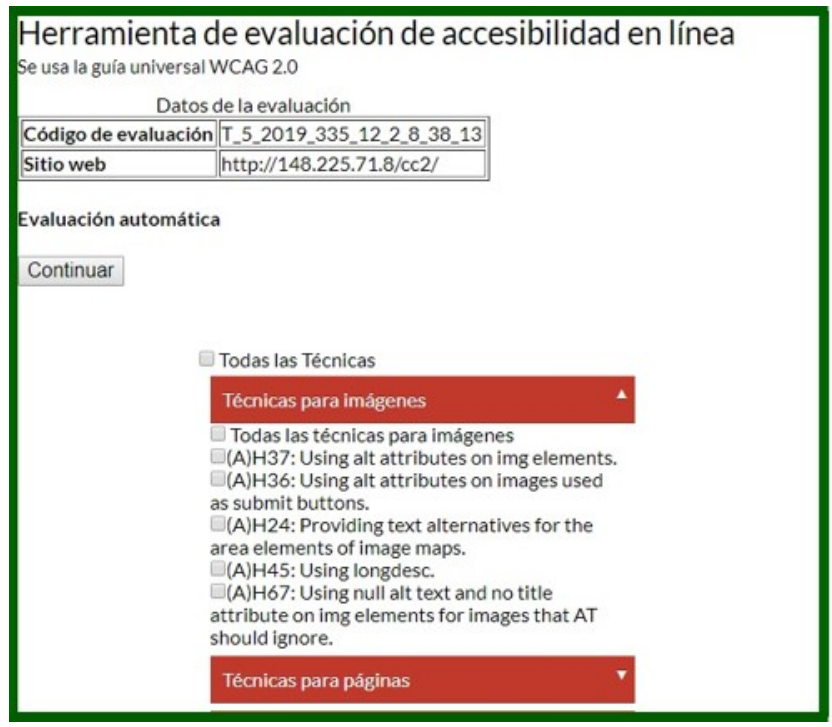

Figura 5: Menú de acordeón para seleccionar técnicas H.

Después de elegir las técnicas, EvA-Web realiza la evaluación de la accesibilidad del sitio web con las técnicas seleccionadas. Se presenta un menú de acordeón, similar al de la Figura 5. en donde sólo se incluyen las categorías que tienen al menos una técnica evaluada. El desarrollador de software puede hacer clic en alguna de las categorías para generar los informes general y detallado de la evaluación. Por ejemplo, al seleccionar el nombre de la categoría imágenes se presenta un informe general similar al de la Figura 6. El informe contiene la 
información de las técnicas evaluadas de la categoría seleccionada (en este ejemplo es la técnica H37 de la categoría Imágenes), la lista de enlaces evaluados, el número de elementos en la página HTML que satisfacen el criterio de la técnica bajo evaluación, la determinación de los elementos que cumplen con los requisitos de la técnicas (advertencia, pasa, no pasa), y el número de renglón en donde se encuentra la etiqueta HTML bajo evaluación. En el caso de la evaluación de conformidad con la técnica H37, advertencia significa que se requiere la intervención humana para validar que la técnica $\mathrm{H}$ se implementó correctamente. Es decir, la etiqueta IMG evaluada tiene el atributo ALT con un texto asignado, el cual podría describir el contenido de la imagen. Sin embargo, las herramientas de evaluación de accesibilidad automática no pueden evaluar si dicha descripción es apropiada para la imagen. Por otra parte, la columna denominada "No pasa" presenta los casos encontrados de la etiqueta IMG que no tienen atributo ALT. La columna "Pasa" presenta los resultados de aquellas técnicas H que no requieren la intervención humana. Por tanto, en el caso de la técnica H37 está reportada con el valor cero.

\begin{tabular}{|c|c|c|c|c|c|c|}
\hline Técnica & Enlace & Flementos & Advertencias & No pasa & Daca & Renalon \\
\hline \multirow[t]{7}{*}{37} & http://148.225.71.8/cc2/ & 10 & 0 & 10 & 0 & \begin{tabular}{|lllllll}
67 & 109 & 112 & 117 & 119 & 124 \\
127 & 173 & 174 & 230 \\
\end{tabular} \\
\hline & new_1.php & 4 & 0 & 4 & 0 & $323277 \quad 197$ \\
\hline & LCC_682.html & 1 & 0 & 1 & 0 & 61 \\
\hline & Unison.html & 32 & 7 & 25 & 0 & \begin{tabular}{|llllll}
201 & 209 & 411 & 419 & 427 & $\stackrel{-}{\square}$ \\
466 & 480 & 494 & 508 & 549
\end{tabular} \\
\hline & Unison_Marco_normativo.html & 14 & 0 & 14 & 0 & $\begin{array}{|llllll|}209 & 562 & 565 & 568 & 571 & \stackrel{1}{\sim} \\
574 & 581 & 582 & 583 & 584\end{array}$ \\
\hline & Unison_Historia_US.html & 15 & 1 & 14 & 0 & $\begin{array}{|llllll|}247 & 464 & 506 & 509 & 512 & \stackrel{1}{-} \\
515 & 518 & 525 & 526 & 527 & \mid\end{array}$ \\
\hline & unison_mx_numeralia.html & 14 & || & 14 & 0 & \begin{tabular}{|lllll}
244 & 529 & 532 & 535 & 538 \\
541 & 548 & 549 & 550 & 551
\end{tabular} \\
\hline
\end{tabular}

Figura 6: Ejemplo de informe resumido para la categoría imágenes de la página web evaluada.

\section{Validación de EvA-Web}

Para validar el prototipo de la herramienta EvA-Web se realizaron pruebas, en el 2019, para determinar que el prototipo identifica correctamente las técnicas $\mathrm{H}$ implementadas. En la primera etapa de la validación, el propósito fue determinar que las técnicas H implementadas en EvA-Web identificaran correctamente los elementos de HTML correspondientes. Para verificar el resultado, se analizaron siete páginas web distintas y, en total, se identificaron manualmente 6,160 elementos de HTML evaluables. La identificación de estos elementos se basó en los resultados de búsqueda que los navegadores de páginas web ofrecen. Además, se accedió al código de la página HTML, que también proporciona el navegador web, para verificar que la técnica $\mathrm{H}$ es aplicable. Por su parte, los resultados de EvA-Web son consistentes 
con la evaluación manual en cada una de las ocho las categorías evaluadas (mencionadas en la Tabla 2).

En la segunda etapa de la validación, se compararon los resultados de la evaluación de accesibilidad del prototipo EvA-Web con herramientas de accesibilidad en línea, Cynthia Says y Taw. La selección de estas herramientas se debe a que los reportes indican el número de línea del código de la página HTML en donde aparece la inconsistencia con la técnica $\mathrm{H}$ evaluada. Para realizar esta actividad, se evaluó otra página web, elegida arbitrariamente, también de la Universidad de Sonora. Ésta corresponde al sitio web de la División de Ciencias Exactas y Naturales (https://dcen.unison.mx/). Se evaluaron únicamente las técnicas $\mathrm{H}$ del nivel A de la WCAG 2.0 (W3C Working Group, 2008) dado que en este nivel se abordan las barreras más comunes a la accesibilidad.

Los resultados parciales de la evaluación se presentan en la Tabla 3. Las celdas de la tabla muestran el número de elementos que se encontraron para cada una de las técnicas $\mathrm{H}$, según la herramienta de evaluación de accesibilidad utilizada. En cuanto a los resultados de Cynthia Says, esta herramienta no encuentra elementos HTML para evaluar la técnica H44, la cual requiere que se use la etiqueta "label" para cada uno de los elementos que conforman al formulario.

Tabla 3: Hallazgos de accesibilidad encontrados por las herramientas EvA-Web, Cynthia Says y Taw.

\begin{tabular}{llll}
\hline Técnica & EvA-Web & Cynthia Says & Taw \\
\hline H02 & 12 & 2 & 1 \\
H37 & 12 & 12 & 4 \\
H24 & 0 & 0 & No determinado \\
H35 & 0 & 0 & No determinado \\
H33 & 0 & 0 & No determinado \\
H36 & 0 & 0 & No determinado \\
H44 & 1 & No encuentra & 1 \\
H45 & 0 & 0 & No determinado \\
H46 & 0 & 0 & No determinado \\
H53 & 0 & 0 & No determinado \\
\hline
\end{tabular}

Las diferencias observadas en los resultados (Tabla 3) se pueden explicar por la forma en que están implementadas las técnicas de evaluación de la accesibilidad y el soporte a distintos lenguajes para el desarrollo de sitios web. De hecho, las comparaciones entre herramientas de evaluación de accesibilidad reportan un amplio rango de variación en cuanto a la salidas incorrectas de herramientas, donde algunas alcanzan alrededor del $30 \%$ de salidas erróneas (Vigo et al., 2013) o violaciones a la accesibilidad no dectectadas porque las herramientas pasan por alto la implementación de técnicas que puedan determinar el grado de implementación de los criterios de éxito, y en promedio sólo se alcanza una cobertura del $50 \%$ de ellos 
(Vigo et al., 2013). Mientras no existan métodos robustos para evaluar la efectividad de estas herramientas se recomienda que el experto en accesibilidad use varias herramientas, dado que algunas de ellas son más efectivas cuando se analizan principios de accesibilidad específicos (Vigo et al., 2013).

Respecto de los resultados que genera EvA-Web, ésta solo revisa el código HTML de la página web y no toma en cuenta el código escrito en lenguaje CSS, JavaScript, entre otros. Esta restricción de capacidad podría explicar las diferencias entre resultados observados. En particular, de las 32 técnicas evaluadas por EvA-Web, Cynthia Says reporta el mismo resultado en 25 de ellas (78\%). Mientras que en el caso de Taw, de las diez técnicas H nivel A que reporta, solo seis muestran el mismo resultado que EvA-Web (60\%). De hecho, el número de técnicas $\mathrm{H}$ que reporta Taw para el nivel A de accesibilidad está por debajo del número de técnicas implementadas en EvA-Web. Por tanto, podemos señalar que los resultados de EvA-Web son consistentes con los resultados de la comparación de herramientas (Vigo et al., 2013) y que existen oportunidades para mejorar el prototipo de la herramienta EvA-Web en términos de efectividad.

En el caso concreto de las diferencias en el reporte de Cynthia Says con respecto de EvAWeb, éstas se presentan en las técnicas: H02, H44, H67, H42, H30 y H65. En el caso de Taw, las diferencias se presentan en las técnicas: H02, H37 y H30. Para entender la diferencia en la aplicación de estas técnicas, se revisó por separado la forma en que cada herramienta de evaluación de accesibilidad determina la aplicación correcta de las recomendaciones. Como ejemplo, presentamos el análisis de la técnica H37.

La técnica H37 trata de mejorar la percepción de los contenidos no textuales: "Cuando una imagen contiene palabras que son importantes para comprender el contenido, el texto alternativo debe incluir esas palabras. Esto permitirá que el texto alternativo juegue la misma función en la página que la imagen" (W3C Working Group, 2012).

Para probar esta técnica (H37), el código HTML de cada etiqueta IMG debe tener el atributo ALT. En el análisis manual de la página bajo estudio, se encontraron doce etiquetas IMG. De éstas, ocho tienen atributo ALT con texto o cadena vacía. Por su parte, la evaluación de Cynthia Says, reporta las doce etiquetas IMG. Además, el reporte indica que cuatro etiquetas no cumplen con la técnica H37. Este resultado es consistente con la evaluación manual. También reporta que seis de esas etiquetas deben ser revisadas manualmente porque el atributo ALT está vacío. Sin embargo, reporta dos casos que corresponden a la técnica H02 en donde la etiqueta IMG debería ser evaluada en el contexto de la etiqueta de enlace $(<a>\ldots<i m g>\ldots</ a>)$ que la contiene.

En el caso de la herramienta Taw, ésta da como resultado cuatro incidencias de errores en el uso de la etiqueta IMG con la técnica H37 porque no contienen el atributo ALT. Por su parte, EvA-Web detecta las doce etiquetas IMG. Al igual que Taw, EvA-Web reporta cuatro casos en dónde la técnica no pasa (etiquetas en las líneas 145, 434, 345, 456). Los restantes son advertencias ya que tienen el atributo ALT y el desarrollador debe verificar manualmente su contenido en las líneas de código: 281, 290, 313, 316, 319, 322, 325 y 328. 


\section{Conclusiones}

En este trabajo se presentaron los resultados de implementar una herramienta de evaluación de accesibilidad que llamamos EvA-Web. El propósito de esta herramienta es identificar los sitios web que hayan implementado las recomendaciones de la WCAG 2.0 en el nivel A. Particularmente, se implementaron las técnicas H para el lenguaje HTML 5.0. Para diseñar la herramienta, se estudió la guía WCAG 2.0 y las técnicas H fueron categorizadas en tablas, formularios, páginas, párrafos, enlaces, estructura, imágenes y otros, para facilitarle al desarrollador de software la selección de las técnicas que le interese evaluar. Además, se desarrolló un web crawler para obtener varios enlaces del sitio web y evaluarlos en un solo paso.

Basándose en los resultados de otras herramientas de evaluación de accesibilidad y las recomendaciones de la literatura, se creó la herramienta con un informe de resultados que identifica con claridad la técnica $\mathrm{H}$ evaluada, el número de línea en donde aparece el código de la etiqueta evaluada, y el valor de la evaluación automática cuando ésta es posible. Además, el desarrollador de software trabaja con un conjunto muy pequeño de interfaces de usuario con el mínimo de datos de entrada. A diferencia de otras herramientas disponibles en línea de forma gratuita, este prototipo permite la evaluación de todas las páginas del sitio web y genera informes en archivos HTML. La herramienta está disponible en español con la intención de que la comunidad de desarrolladores hispanohablantes disponga de otro instrumento para evaluar la accesibilidad.

Como trabajo futuro se tomarán en cuenta las capacidades que tienen las herramientas evaluadas para mejorar el prototipo EvA-Web. Algunas herramientas tienen implementado una opción de corrección de la anomalía encontrada. Además, es importante proporcionar al desarrollador de software mecanismos que le permitan verificar que la técnica H se implementó apropiadamente. El diseño de estas dos características requiere de la comunicación con los desarrolladores de sitios web para conocer las prácticas que aplican para liberar sitios web accesibles. Además, la construcción del prototipo de EvA-Web está conceptualizado en el contexto de un proyecto mayor, en donde la herramienta se podría convertir en un componente principal en el diagnóstico de barreras de accesibilidad, como apoyo a la introducción de un proceso de mejora de la calidad de los sitios y aplicaciones web.

A futuro se estudiarán e implementarán otras técnicas de la WCAG 2.0 orientadas a tecnologías como CSS o JavaScript. Finalmente, el desarrollo de una herramienta robusta para la evaluación de la accesibilidad puede servir para que las empresas, y otras organizaciones, analicen la accesibilidad de sus sitios web y verifiquen si sus contenidos están llegando a todos sus usuarios. 


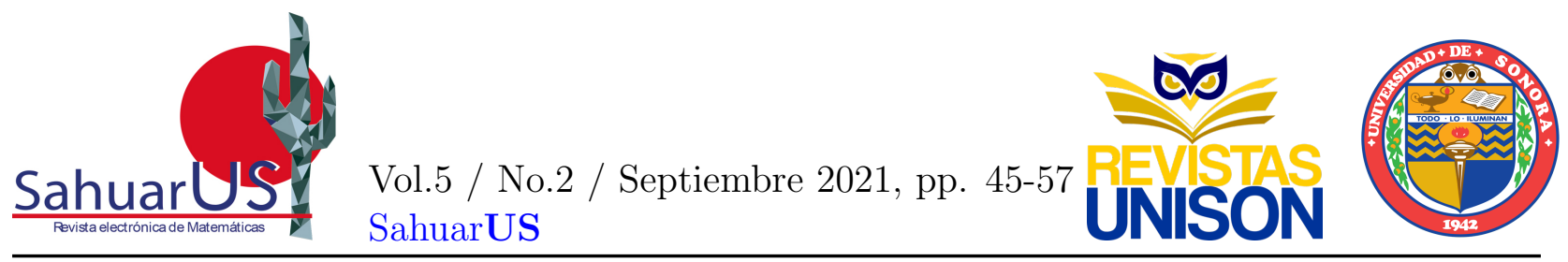

\section{Referencias}

Acosta, T., Acosta-Vargas, P., and Luján-Mora, S. (2018). Accessibility of eGovernment Services in Latin America. In 2018 International Conference on eDemocracy $\& 3$ eGovernment (ICEDEG), pages 67-74. IEEE.

Agangiba, M. and Kabanda, S. (2017). Research foci, methodologies, and theories used in addressing E-government accessibility for persons with disabilities in developing countries. Interdisciplinary Journal of Information, Knowledge, and Management, 12:245-268.

Henry, S. L. and McGee, L. (2018). Accessibility. https://www.w3.org/standards/ webdesign/accessibility. Consultado el 10 de agosto de 2018.

Instituto Nacional de Estadística y Geografía (México) (2016). La discapacidad en México, datos al 2014. http://conadis.gob.mx/gob.mx/transparencia/transparencia_ focalizada/La_Discapacidad_en_Mexico_datos_2014.pdf. Consultado el 10 de agosto de 2018 .

Laitano, M. I. (2015). Accesibilidad web en el espacio universitario público argentino. Revista española de documentación científica, 38(1):e079-e079.

Power, C., Freire, A., Petrie, H., and Swallow, D. (2012). Guidelines are only half of the story: accessibility problems encountered by blind users on the web. In Proceedings of the SIGCHI conference on human factors in computing systems, pages 433-442.

Vigo, M., Brown, J., and Conway, V. (2013). Benchmarking web accessibility evaluation tools: measuring the harm of sole reliance on automated tests. In Proceedings of the 10th International Cross-Disciplinary Conference on Web Accessibility, pages 1-10.

W3C Working Group (2008). Web Content Accessibility Guidelines (WCAG) 2.0. https: //www.w3.org/TR/WCAG20/. Consultado el 10 de agosto de 2018.

W3C Working Group (2012). HTML and XHTML Techniques for WCAG 2.0. https: //www.w3.org/TR/WCAG20-TECHS/html.html. Consultado el 23 de noviembre de 2019.

W3C Working Group (2016). Techniques for WCAG 2.0: Techniques and Failures for Web Content Accessibility Guidelines 2.0. https://www.w3.org/TR/WCAG20-TECHS/, Consultado el 10 de agosto de 2018.

W3C Working Group (2018). Web Accessibility Initiative (WAI). Introduction to web accessibility. https://www.w3.org/WAI/fundamentals/accessibility-intro/, Consultado el 10 de agosto de 2018 .

World Health Organization (2011). World Report on Disability. http://www.who.int/ disabilities/world_report/2011/report.pdf. Consultado el 10 de agosto de 2018. 